\title{
Interstellar and Solar System organic matter preserved in interplanetary dust
}

\author{
Scott Messenger and K. Nakamura-Messenger \\ NASA Johnson Space Center, Houston, Texas, USA \\ email: scott.r.messenger@nasa.gov
}

\begin{abstract}
Interplanetary dust particles (IDPs) collected in the Earths stratosphere derive from collisions among asteroids and by the disruption and outgassing of short-period comets. Chondritic porous (CP) IDPs are among the most primitive Solar System materials. CP-IDPs have been linked to cometary parent bodies by their mineralogy, textures, C-content, and dynamical histories. CP-IDPs are fragile, fine-grained $(<\mathrm{um})$ assemblages of anhydrous amorphous and crystalline silicates, oxides and sulfides bound together by abundant carbonaceous material. Ancient silicate, oxide, and $\mathrm{SiC}$ stardust grains exhibiting highly anomalous isotopic compositions are abundant in CP-IDPs, constituting $0.01-1 \%$ of the mass of the particles. The organic matter in CP-IDPs is isotopically anomalous, with enrichments in $\mathrm{D} / \mathrm{H}$ reaching $50 \mathrm{x}$ the terrestrial SMOW value and $15 \mathrm{~N} / 14 \mathrm{~N}$ ratios up to $3 \mathrm{x}$ terrestrial standard compositions. These anomalies are indicative of low $\mathrm{T}(10-100 \mathrm{~K})$ mass fractionation in cold molecular cloud or the outermost reaches of the protosolar disk. The organic matter shows distinct morphologies, including sub-um globules, bubbly textures, featureless, and with mineral inclusions. Infrared spectroscopy and mass spectrometry studies of organic matter in IDPs reveals diverse species including aliphatic and aromatic compounds. The organic matter with the highest isotopic anomalies appears to be richer in aliphatic compounds. These materials also bear similarities and differences with primitive, isotopically anomalous organic matter in carbonaceous chondrite meteorites. The diversity of the organic chemistry, morphology, and isotopic properties in IDPs and meteorites reflects variable preservation of interstellar/primordial components and Solar System processing. One unifying feature is the presence of sub-um isotopically anomalous organic globules among all primitive materials, including IDPs, meteorites, and comet Wild-2 samples returned by the Stardust mission. We will present an overview of the current state of understanding of the properties and origins of organic matter in primitive IDPs.
\end{abstract}

Keywords. 